\title{
First order gravity on the light front
}

\section{Sergey Alexandrov*}

Laboratoire Charles Coulomb, Université Montpellier 2

E-mail: salexandeuniv-montp2.fr

\begin{abstract}
After a brief review of unusual features of the light front canonical formulations of field theories, I present the analysis of the canonical structure of the first order formulation of general relativity on a lightlike foliation. It appears to be quite different from the usual spacelike case leading, for instance, to the presence of tertiary constraints. Besides, I discuss the issue of the zero modes and argue that there might be some hidden correspondence with two-dimensional theories.
\end{abstract}

Frontiers of Fundamental Physics 14 - FFP14,

15-18 July 2014

Aix Marseille University (AMU) Saint-Charles Campus, Marseille

\footnotetext{
* Speaker.
} 